\title{
IdeAs
}

Idées d'Amériques

\section{La paix selon les femmes autochtones}

\section{Anna Schmit}

\section{(2) OpenEdition}

Journals

Édition électronique

URL : https://journals.openedition.org/ideas/1848

DOI : $10.4000 /$ ideas. 1848

ISSN : 1950-5701

Éditeur

Institut des Amériques

\section{Référence électronique}

Anna Schmit, "La paix selon les femmes autochtones », IdeAs [En ligne], 9 | 2017, mis en ligne le 03 juillet 2017, consulté le 18 octobre 2022. URL : http://journals.openedition.org/ideas/1848; DOI :

https://doi.org/10.4000/ideas.1848

Ce document a été généré automatiquement le 18 octobre 2022.

\section{(c) (i) ()}

Creative Commons - Attribution - Pas d'Utilisation Commerciale - Pas de Modification 4.0 International - CC BY-NC-ND 4.0

https://creativecommons.org/licenses/by-nc-nd/4.0/ 


\title{
La paix selon les femmes autochtones
}

\author{
Anna Schmit
}

En septembre 2012, au même moment où le président Santos annonce l'ouverture de négociation de paix avec les Farc, les femmes autochtones de Colombie se sont alliées pour produire un plaidoyer international devant le Comité des Nations unies pour l'élimination des discriminations envers les femmes (COEDEF) ${ }^{1}$, garant des droits inscrits dans la Convention du même nom $(\mathrm{CEDEF})^{2}$. Cet article a pour ambition d'analyser la formalisation d'un discours politique produit des femmes du mouvement autochtone et qui proclame la centralité des droits sexuels et reproductifs dans la construction de la paix. Ayant tout d'abord débuté leur action par la mise en relief de leur appartenance ethnique, et ce dans le but d'insérer leur revendication dans le cadre multiculturel colombien, ces femmes ont finalement questionné les rapports sociaux de sexe prévalant dans leurs sociétés (autochtone et colombienne) pour envisager les manières de les transformer avec le soutien de l'État colombien. Les débats ayant nourri leur réflexion ont débouché sur un positionnement politique courageux, affirmant que la paix sociale commence sur le terrain sexuel, entendu comme l'espace intime dans lequel elles exercent leur sexualité et dans lequel elles tissent leur intimité avec les hommes qui leur sont proches. Elles ont ainsi remis en question le " dispositif

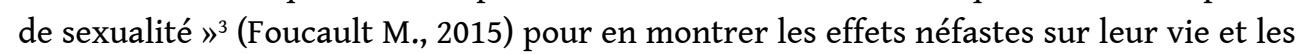
risques qu'il représentait, non seulement pour la construction de la paix, mais aussi pour les revendications autochtones ${ }^{4}$. L'enjeu de ces luttes en effet est l'autodétermination des peuples sur leurs territoires (Gros C., 2010), lesquels sont conçus comme les espaces d'exercice d'une souveraineté politique, déterminant leur possibilité de reproduction sociale et culturelle (Bellier I., 2013). De ce fait, la permanence des peuples autochtones sur leur territoire mérite une protection juridique particulière, actuellement mise à mal par l'intrusion des acteurs armés (armée nationale, paramilitaire, guérilla) et par la prolifération des projets miniers de grande ampleur. 


\section{Les femmes autochtones et le multiculturalisme constitutionnel}

2 La Constitution colombienne de 1991 enjoint l'État à protéger la diversité culturelle de la nation et introduit la reconnaissance de droits collectifs aux peuples autochtones. Cette reconnaissance passe notamment par la constitution d'une gouvernance autonome circonscrite à leur territoire (Gros C., 2010). De plus, elle revendique l'égalité des sexes et reconnaît aux femmes des droits spécifiques en les protégeant des violences et des discriminations commises à leur encontre. Elle se porte par ailleurs garante de leurs droits sexuels et reproductifs. Enfin, elle a transformé le système de participation démocratique en introduisant légalement l'obligation d'adapter les politiques publiques aux catégories sociales qui en bénéficient et de les élaborer en concertation avec elles. Des espaces de participation démocratique se sont par conséquent ouverts aux " peuples autochtones » et aux «femmes », et accueillent leurs représentant.e.s pour négocier avec l'État l'élaboration et la mise en place des politiques publiques les concernant. Cependant, malgré ces avancées, les normes juridiques promulguées dans cette nouvelle Constitution ont conduit à un cloisonnement tacite séparant les catégories sociales relevant des " droits des femmes » et celles relevant des «droits des peuples autochtones». Cette nouvelle normativité a eu en réalité pour effet de restreindre la protection des femmes autochtones qui ont vu par ailleurs leur participation au jeu politique colombien bloquée et leurs conditions de vie se dégrader à cause de l'intensification de la violence armée et de la prolifération minière sur leurs territoires. C'est dans le souci de remédier à cette dégradation et de décloisonner la normativité du multiculturalisme constitutionnel que naît le premier plaidoyer des femmes autochtones devant le CoEDEF, au moment où le gouvernement colombien et les FARC entament des pourparlers de paix. Ce plaidoyer s'inscrit par conséquent dans la dynamique nationale stimulant des réflexions collectives sur ce qu'est la paix et comment y parvenir.

\section{Cibler les violences}

3 L'action débute en septembre 2012 quand la Fuerza de Mujeres Wayúu, - association locale de défense des droits des peuples autochtones issue d'un mouvement de victimes des crimes paramilitaires formé en 2007 dans La Guajira - invite des femmes autochtones "leaders", à se former aux principes de la CEDEF, à réfléchir aux modalités que pourraient prendre l'application du texte onusien dans les contextes autochtones et à poser la nécessité de rédiger un contre-rapport ${ }^{5}$, recensant les violences spécifiques dont elles sont les cibles.

L'enjeu du plaidoyer des femmes autochtones a été de rendre visibles les différents types de violence qu'elles affrontent dans leur quotidien, semblables à celles qui ont déjà été exposées en 1995 lors de la Conférence des femmes à Pékin (Bellier I.,1995). Au début des années 2010 en Colombie, les femmes autochtones en effet considèrent subir une violence qui non seulement émane du conflit armé mais aussi qui se loge dans les rapports qu'elles entretiennent avec leurs conjoints dans la sphère intime. Une de leurs priorités a donc été d'intégrer au document final l'articulation des violences sexuelles perpétrées par les acteurs armés (réguliers ou non) avec celles commises dans les communautés autochtones et d'exiger de l'État une intervention visant à les faire 
cesser. En témoignant des violences commises contre elles au sein même de leur communauté, elles ont engagé une rupture avec la conception d'une tradition immuable dont elles seraient les " gardiennes ».

5 Ces femmes se sont efforcées de montrer comment le conflit armé et l'exploitation minière diffuse dans leur quotidien une violence sociale, armée et sexuelle. Selon elles, les acteurs armés accompagnent l'usurpation et la spoliation de terres orchestrées par l'industrie d'extraction minière. Ils la facilitent en établissant un climat de peur qui parvient à paralyser l'activisme politique des hommes et des femmes autochtones. L'implantation minière aux abords (ou dans) les territoires autochtones et la proximité des soldats (réguliers ou non) avec les communautés indiennes entrainent ainsi une augmentation de la prostitution et des viols perpétrés à l'encontre de jeunes filles. Ce faisant, les grossesses non désirées augmentent, tout comme les risques de monoparentalité contrainte. Celle-ci déstabilise l'équilibre social dans les communautés et met à mal la survie des peuples. Selon l'ONG Sisma Mujer (organisation féministe colombienne), les violences sexuelles contre les femmes sont significatives de l'imposition d'un modèle de société dans lequel la construction sociale de la différence des sexes exacerbe la relation de subordination qui lie les femmes aux hommes en restreignant la liberté sexuelle des premières. Ces pratiques visent à construire un " dispositif de sexualité » hégémonique balisant un exercice discipliné de la sexualité des femmes, qui, répondant aux critères "traditionnels et stéréotypés " des rapports sociaux de sexes, les oblige à développer une sexualité passive (Sisma mujer, 2009).

6 Les femmes autochtones se sont appuyées sur la dénonciation qu'elles faisaient des dynamiques belliqueuses et industrielles pour mettre en cause l'agencement des rapports sociaux de sexes dans leurs sociétés. En effet, elles rendent le conflit armé responsable du durcissement de ces rapports au sein de leur peuple. Cela leur a permis de porter une critique, assez inédite, de cette idée de "tradition " suivant laquelle les femmes ne pouvaient disposer librement de leur corps. Ces femmes ont clairement dénoncé les cas de mariages forcés, engageant fréquemment de très jeunes filles avec des hommes bien plus âgés, les relations incestueuses et les viols, la violence des "rites " comme les mutilations génitales pratiquées de façon très minoritaire dans certaines communautés, ou encore leur manque d'accès à une justice prenant réellement en compte leurs problématiques. Elles ont par ailleurs fréquemment dénoncé la sommation qui leur est faite de ne pas participer activement à un acte sexuel et l'interdiction d'érotiser leur désir sexuel. Ces formes de contrôle de la libido féminine s'inscrivent ainsi selon elles dans la continuité d'un ordre social particulier, façonné par les logiques du conflit armé, et dans lequel se reflète l'objectivation de leur corps.

7 Les femmes autochtones ont mis en relief le lien existant entre la violence publique manifeste du climat de guerre avec la violence privée qui s'exerce à leur encontre dans leur communauté. Dans les deux cas, elles l'interprètent comme une violence sexuelle façonnant un "dispositif de sexualité » à l'œuvre dans les sociétés autochtones et renforcé par les logiques du conflit armé. Le message adressé aux peuples autochtones est que la défense du territoire ne peut commencer que par la défense du corps des femmes, si celui-ci n'est pas protégé, alors le territoire ne peut l'être non plus et les peuples ne peuvent être souverains. Le message adressé à l'État colombien est que le respect de leurs droits sexuels et reproductifs par tous les hommes (armés, 
fonctionnaires, paysans ou artisans, qu'ils soient autochtones ou non) est la condition sine qua non à l'avènement d'une paix durable.

\section{Les droits sexuels et reproductifs et la paix}

8 L'action des femmes autochtones devant le CoEDEF témoigne d'un effort pour articuler multiculturalisme et féminisme dans l'émergence de la paix. C'est en assumant la position politique des peuples autochtones, tournée vers l'auto-gouvernance et dans la défense du territoire (Benavides C. et Duarte C., 2010 ; Gros C., 2010; Mazars N., 2013, Bellier I., 2013), qu'elles ont pu affirmer l'existence d'un lien entre la paix et leur capacité à «disposer d'elles-mêmes » (Schmit A., 2016). Elles ont posé dans le débat public une critique acerbe d'un dispositif de sexualité dans lequel la sexualité des femmes demeure assujettie à celle des hommes et elles ont réussi à montrer sa perméabilité à l'hégémonie d'un ordre social, gouverné par les logiques du conflit armé et de ses complexes militarisés. Selon elles, les violences commises par les acteurs du conflit et celles commises dans leur communauté se légitiment mutuellement.

Ces réflexions ont amené les femmes autochtones à adopter un positionnement politique spécifique selon lequel la construction de paix sociale ne peut advenir que par une sérieuse prise en compte de leurs droits sexuels et reproductifs qui doit impliquer une remise en cause profonde du système de genre prévalant en Colombie et dans les sociétés autochtones. Or les accords, signés entre le gouvernement de Santos et les FARC le 26 novembre 2016 incluent de manière pionnière une perspective de genre, et ce malgré les fortes résistances que cette inclusion a suscitées dans certains milieux conservateurs et catholiques. En effet, ces accords ont fait l'objet de fortes oppositions qui ont abouti à leur rejet, lors du référendum du 2 octobre 2016, ce qui a obligé les parties belligérantes à les ajuster. Or si la deuxième version de ces accords pointe à plusieurs reprises les violences sexuelles commises contre les femmes (autochtones ou non) dans le cadre du conflit armé et montrent un réel effort pour penser une réparation effective et efficace pour les victimes, ils peinent cependant à en mesurer les causes structurelles et à en identifier les effets dans la sphère privée. Hormis une évocation succincte de la protection sociale qu'il conviendrait d'offrir aux «femmes rurales» (p. 33), catégorie englobant, selon les signataires, celles de "femmes autochtones ", ils ne situent aucunement les droits sexuels et reproductifs comme des impératifs dans la construction de la paix, laissant ainsi douter d'une réelle prise en compte de la parole des «femmes concernées».

\section{BIBLIOGRAPHIE}

Références

BELLIER Irène, « Les femmes indigènes : double lutte pour la survie, Vivre Autrement, Conférence des Femmes - Pékin 1995. 
BELLIER Irène, Terres, territoires, Ressources, Paris : L'Harmattan, 2014, Collections horizons autochtones, SOGIP.

BENAVIDES Carlos, DUARTE Carlos, « Descentralización, autonomía, trasferencias y gobierno propio en Colombia» pp. 185-233 in FOYER Jean et GROS Christian (eds), Desarrollo con identidad? Gobernanza económica indígena, siete estudios de caso, Lima : IFEA Acte et Mémoire, 2010.

FOUCAUlT Michel, Histoire de la sexualité I La volonté de savoir, Paris: Gallimard, nrf, 2015, 1e édition: 1976.

GROS Christian, Nación, Identidad y violencia, el desafío latinoamericano, Bogotá : Universidad Nacional de Colombia, Universidad de los Andes, Instituto Francés de estudios andino, 2010.

MAZARS Nadège, Les ruses de la pratique subalterne. La santé gérée par les autochtones en Colombie, un multiculturalisme de domination et/ou d'autonomie?, Thèse de doctorat en sociologie, IHEAL, 2013.

schmit Anna, Teñiamos el sueño de cambiar el mundo. Expérience militante de femmes autochtones de La Guajira - Colombie. Thèse de doctorat en anthropologie sociale, EHESS, 2016.

SISMA MUJER, Mujeres en conflicto : violencia sexual y paramilitarismo, 2009.

\section{NOTES}

1. Ce Comité est composé de vingt-deux expert.e.s qui examinent tous les quatre ans l'évolution de la situation des femmes dans les Etats qui lui sont parties, en se basant sur les rapports que ces derniers produisent et sur les contre-rapports formulés par des organisations de la société civile. Mon propos se base sur l'examen de la Colombie réalisé par le CoEDEF en octobre 2013.

2. Cet article s'appuiera sur l'ethnographie que j'ai réalisée de l'élaboration du plaidoyer des femmes autochtones et restituera quelques éléments des débats l'ayant animé, entre 2012 et 2013 (Schmit A., 2016).

3. Le "dispositif de sexualité" se réfère à une mise en discours de la sexualité, produisant des rapports de pouvoir, se logeant dans des relations interpersonnelles et ayant des effets concrets sur la construction de soi (Foucault M., 2015).

4. Selon le recensement des populations ethniques, effectué par le DANE en 2005, la Colombie abriterait 87 peuples autochtones regroupant 1378884 personnes.

5. Contre rapport qualifie les documents élaborés par des organisations de la société civile et transmis aux Comités onusiens. Il vise donc à contrebalancer les rapports officiels produit par les Etats.

\section{AUTEUR}

\section{ANNA SCHMIT}

Docteure en anthropologie sociale, associée au IIAC-LAIOS/EHESS 